\title{
Spielarten geistlicher Trauer. Maria Magdalena am Grab. Teil II: \\ Deutsche Predigten und Prosa-Übersetzungen des Ps.-Origenes-Textes ${ }^{1}$
}

\author{
Freimut Löser \\ Universität Augsburg \\ freimut.loeser@phil.uni-augsburg.de \\ https://dx.doi.org/10.12795/futhark.2011.06.06
}

\begin{abstract}
Der Gesamtbeitrag gilt der Trauer Maria Magdalenas am Grab (Johannes 20,11-18), die zwischen karfreitäglicher Verzweiflung und österlichem Trost schwankt.

Teil I (Futhark Nr. 4): Ausgangspunkt (I.) ist die (aus dem späten 12. oder frühen 13. Jahrhundert stammende) lateinische Predigt des Ps.-Origenes, die in Europa weite Verbreitung und zahlreiche Bearbeitungen fand. Die deutschen poetischen Bearbeitungen (II: 'Der Saelden Hort', Heinrichs von Neustadt 'Von Gottes Zukunft', 'Bairische Magdalenenklage') stellen die Heldin in den Mittelpunkt und akzentuieren den Trauer-Diskurs im Sinne öffentlich sichtbarer Gebärden der Trauer.

Teil II: Die genuinen deutschen Predigten (III. Meister Eckhart, Marquard von Lindau) eröffnen den Horizont der Mystik (Einung mit Gott sunder mittel), eine lateinische Predigt des tschechischen Reformers Milič von Kremsier - auch in deutscher Übertragung überliefert - stellt den Apell gegen Sünde und für ein gottgefälliges Leben in den Vordergrund. Die deutschen Prosaübersetzungen des 15. Jahrhunderts (IV.) entstehen im Zusammenhang der Klosterreformen (anonymer dominikanischer [?] Nürnberger 'Magdalenenlibellus', der Kartäuser Heinrich Haller, die Zisterzienserin Regula, der benediktinische Laienbruder Lienhart Peuger) und beziehen die Situation auf die Situation im Kloster. Ein letzter Punkt (V.) bietet eine Zusammenschau der Entwicklungsmöglichkeiten des Textes und der 'Spielarten geistlicher Trauer'.
\end{abstract}

Keywords: Ps.-Origen - Predigten - 'Der Saelden Hort' - Heinrich von Neustadt - Bairische Magdalenenklage - Mystik - Meister Eckhart - Marquard von Lindau - Jan Milič von Kremsier - Heinrich Haller, Regula, Lienhart Peuger Nürnberger 'Magdalenen-libellus'.

${ }^{1}$ Aus technischen Gründen erscheint der Aufsatz in zwei Teilen. 
Abstract: The article as a whole deals with the grief of Mary Magdalen at the empty tomb (John 20,11-18).

Part one (Futhark 4): A latin sermon, (wrongfully) attributed to Origen during the Middle Ages discusses her attitude of grief, mourning and sorrow and the possibility of consolation. Widely spread in Europe (there is a lost version of Chaucer's) the sermon was repeatedly revised, used, translated in several German versions: The poetic revisions ('Der Saelden Hort', Heinrichs von Neustadt 'Von Gottes Zukunft', 'Bairische Magdalenenklage') put Mary in the center. She now is a heroine in the sense of courtly novels or epics; grief and mourning change to visible acts.

Part Two: The German sermons (of Meister Eckhart's and the Franciscan Marquard's of Lindau) open up the context of Mysticism (becoming one with God without means); a latin sermon of the čech reformer Jan Milič (also translated into German) uses the example to vote against sin. The numerous German prose translations belong into the monastic traditions of different orders and transfer the text to the monastic discipline of nuns and laybrothers.

Keywords: Grief of Mary Magdalen at the empty tomb (John 20,11-18) consolation - Ps.-Origen - sermon - German revisions (poetic, prose) - 'Der Saelden Hort' - Heinrich von Neustadt - Bairische Magdalenenklage - Mystics Meister Eckhart - Marquard von Lindau - Jan Milič von Kremsier - Monastic Reform (Heinrich Haller, Regula, Lienhart Peuger) - Nürnberger 'Magdalenenlibellus'.

Nachdem im ersten Teil des Aufsatzes die Pseudo-OrigenesHomilie über Maria Magdalena am Grab vorgestellt und ihre deutschen poetischen Bearbeitungen besprochen wurden, soll es hier um die Rezeption in der Predigt (Abschnitt III) und die Prosaübersetzung (IV) gehen, sowie ein Gesamtresümée versucht werden (V). Die Wirkungsgeschichte der Pseudo-Origenes-Homilie im Bereich der Prosa (hier der Predigt) ist im Deutschen durch zwei Namen geprägt: Meister Eckhart und Marquard von Lindau. Dazu kommen für das Lateinische Jan Milič von Kremsier und für das Niederländische Jan Storm. ${ }^{2}$

2 Zu Storm: Bram Rossano: Die deutschen und niederländischen Bearbeitungen der Pseudo-Origenes-Magdalenenklage, in: Beiträge zur Geschichte der deutschen Sprache und Literatur 126 (2004), S. 233-260; bes. S. 245-250. 
III.1.

Eckhart hat sich in zwei Predigten dem entsprechenden Bibeltext gewidmet. Über die Predigt Nr. 55 der kritischen Edition berichten Handschriften und frühe Drucke einleitend, sie gehöre Vff sant Marie magdalene tag, obwohl man das euangelium [...] nach gewonlichem brauch der kirchen vff Donnerstag in Osterfeyertagen liset. ${ }^{3}$ Predigt 56 ist nur in der bekannten Sammlung des 'Paradisus anime intelligentis' überliefert. Sie ist dort am Donnerstag nach Ostern eingeordnet. ${ }^{4}$ Die Verfasserschaft Eckharts ist für beide Predigten gesichert. Und doch fallen sie aus dem Rahmen dessen, was wir von ihm kennen und erwarten. Das hat eine einfache Erklärung. Der Herausgeber Quint hat davon gesprochen, dass sich Eckhart in beiden Predigt-texten der Homilie des Pseudo-Origenes anschließe oder diese benutze. ${ }^{5}$ Dies ist untertrieben, denn Eckhart gibt zwar gekürzt und bearbeitet den PseudoOrigenes Text im Wesentlichen einfach wieder und in Predigt 55 ist nur ein Abschnitt über Maria Magdalenas Verhalten am Grab als eigenständig zu werten: Hier behandelt Eckhart ausführlich die Frage, war umbe si stuont und niht ensaz. ${ }^{6}$ Aber auch in diesem Punkt formuliert er die Antwort in seiner volkssprachlichen Predigt nicht neu und genuin, er greift vielmehr auf Gedanken und Definitionen über Stehen und Sitzen aus seinem Johanneskommentar (zu den entsprechenden Bibelversen) zurück. ${ }^{7}$ Dort hatte er zu Jo 20,11 auch ausdrücklich vermerkt: Verba ista usque ibi: 'cum ergo sero essent [Jo 20,19], Gregorius et specialiter Origenes in Homilia pulchre, diffuse et

\footnotetext{
${ }^{3}$ Die kritische Ausgabe der deutschen und lateinischen Werke Meister Eckharts wird zitiert nach Meister Eckhart: Die deutschen und lateinischen Werke. Hg. im Auftrag der Deutschen Forschungsgemeinschaft. J. Quint (Hg.), Die deutschen Werke, Bd. I-III und V, Stuttgart 1958-1975; Bd. IV hg. unter Mitarbeit von W. Klimanek und F. Löser von G. Steer, Stuttgart 1998ff. (zit. als DW I-V); E. Benz/K. Christ/B. Decker/H. Fischer /J. Koch/L. Sturlese/K. Weiss/A. Zimmermann (Hgg.), Die lateinischen Werke Bd. I-V, Stuttgart 1956ff. (zit. als LW I-V), hier zitierte Stelle: DW II, S. 572 und 575, Variante zu Z.1.

${ }^{4}$ DW II, S. 586f.; vgl. Philipp Strauch (Hg.), Paradisus anime intelligentis (DTM 30), Berlin 1919 , S. $61 \mathrm{Nr} .26$.

${ }^{5}$ DW II, S. 574 und 587

${ }^{6}$ DW II, S. $580 f$.

${ }^{7}$ LW III, n. 699-706, S. 613-619.
} 
devote valde exponit'. ${ }^{8}$ Eckhart sieht Origenes also auch in seinem lateinischen Johanneskommentar als vorbildhaft an. Eckhart folgt der Homilie des Pseudo-Origenes in seiner Predigt 55 nicht genau und in allen Details: Vielmehr greift er nur einzelne Fragen heraus. Diese beantwortet er allerdings nicht eigenständig, sondern im selben Sinn wie die Vorlage: War umbe sprach si: „zeige mir, war dû in getragen hâst; ich wil in dâ nemen?" H\#te er in getragen in des rihters hûs, wölte si in ouch dâ genomen hân? „Jâ", sprach ein meister [gemeint ist natürlich PseudoOrigenes], „si $h \nRightarrow$ \#te in genomen in des rihters slozze“. ${ }^{9}$ Ähnlich eng lehnt sich Eckhart auch in der Frage, warum sich Maria Magdalena nicht fürchtete, an seine Vorlage an. Auch hier wird die Antwort (sie hatte nichts zu verlieren, denn sie hatte sich ganz hingegeben, wan si sîn was, wan ir herze mit im was) ${ }^{10}$ als Antwort des meisters Origenes gekennzeichnet. Aber auch die folgende Aussage Eckharts, dass Maria Magdalena begehrte, daz ir sêle in dem grabe gewonet $h \# t e$ und ir lîchame bî dem grabe: ir sêle inne und ir lîchame dâ bî ${ }^{11}$, entstammt, was der Herausgeber Quint übersah, Eckharts Vorlage; das gilt ebenso für das Motiv der Furcht Maria Magdalenas, Christi Grab auch noch zu verlieren. Schon hier sieht man, welches Themenspektrum Eckhart aus dem 'Angebot' der Quelle auswählt: Nicht den Trauer-, sondern den Einheitsdiskurs: Maria sucht den Herrn, will „bei inm wohnen“ (übertragen auch im Tod) und will ihn nicht verlieren. Auch die Betonung der Demut als wichtigster Tugend entnimmt Eckhart seiner Quelle genauso wie den Schluss seiner Predigt über die Tatsache, dass Maria den Herrn nicht erkannte. ${ }^{12}$ Wie weit die Abhängigkeiten im Einzelnen gehen, habe ich andernorts demonstriert. ${ }^{13}$ Diese Vorlagentreue betrifft

${ }^{8}$ LW III, n. 699, S. 613. Überhaupt verwendet Eckhart diese Pseudo-Origenes-Homilie häufiger, auch in seinen lateinischen Sermones. Vgl. LW IV, S. 81, Z. 4f. und Anm. 4 und besonders ebd., S. 31f., Z. 14-2 und S. 32 Anm. 1.

${ }^{9}$ DW II, S. 577,1-3.

${ }^{10}$ DW II, S. $577 f$.

11 DW II, S. 579,1-2.

12 DW II, S. 579, 581 und 583f.

${ }^{13}$ Freimut Löser: Jan Milíč in europäischer Tradition. Die Magdalenen-Predigt des PseudoOrigenes, in: Dominique Fliegler/Václav Bok (Hgg.): Deutsche Literatur des Mittelalters in Böhmen und über Böhmen. Vorträge der internationalen Tagung, veranstaltet vom Institut für Germanistik der Pädagogischen Fakultät der Südböhmischen Universität České Budějovice 1999, Wien 2001, S. 225-246; hier S. 231. 
vor allem auch die Bewertung der minne Maria Magdalenas und Christi (jeweils Genitivus subjectivus und objectivus). So sagt Eckhart:

1 Ein meister sprichet: herre, waz meinest dû dâ mite, daz dû dich dirre vrouwen sô lange mohtest entziehen; wâ mite hât si ez verschult, oder waz hât si getân? Sît dem mâle, daz dû ir ir sünde verg\#be, sô enhât si niht getân, wan daz si dich minnete. H\#te si iht getân, daz vergip ir durch dîne güete. Minnete si

5 dînen lîp, dô weste si doch wol, daz diu gotheit dâ bî was. Herre, ich spriche dir an dîne götlî́che wârheit, daz dû hâst gesprochen, dû würdest ir niemer benomen. Dû hâst wâr, wan dû k\#me ûz irm herzen nie und spr\#che: 'der dich minnete, den wellest dû wider minnen, und der dâ vruo ûf ist, dem wellest dû erschînen'.14

Quint hat es vermieden, das Zitat, das durch den Hinweis Ein meister sprichet eingeleitet wird, zum Ende hin abzugrenzen. Als Leser vermutet man (ausgehend vom Gegensatz „Ein meister-Ich“) einen Neu-Ansatz in Z. 5 mit "Herre, ich spriche..." Ein genauer Blick in die Pseudo-OrigenesPredigt zeigt aber, dass es sich bei diesem 'Ich' des Predigers, das für Maria Magdalena vehement Partei ergreift und dabei kühn den Auferstandenen anspricht (Herre, ich spriche dir an dîne götlîche wârheit), keineswegs um Eckhart handelt, sondern dass er auch hier nur seiner Vorlage folgt. Diese Vorlage betrifft nun auch die Bewertung der Trauer durch Eckhart. Magdalenas Klage wird ausgelöst durch den Verlust des körperlich-menschlichen Christus; und doch weiß sie immer um die Einheit von Mensch und Gott. Ihre Liebe gilt dem Einen. Und deshalb muss (so äußert sich Eckhart immer und deshalb entspricht die Pseudo-Origenes-Predigt so genau seinem Denken) - deshalb mus s Gott die Liebende lieben. Daraus kann Trost (der einzige Trost) erwachsen. Letztlich konsequent ergibt sich aus alldem aber auch ein Eingriff in die Vorlage. ${ }^{15}$ Nur in einem entscheidenden Punkt nimmt

${ }^{14}$ DW II, S. 582,5-12.

${ }^{15}$ Vgl. Adolf Patera: Hradecký Rukópis, Praha 1881, S. V-XI (zur Handschrift) und S. 77144 (Textabdruck), S. 441, sl. 242 ${ }^{\mathrm{b}}-243^{\mathrm{a}}$. 
Eckhart diese Umwertung und Neu-Definition vor: Als Maria Magdalena im Grab den beiden Engeln begegnet, da versucht die Pseudo-OrigenesHomilie sie vom tröstenden Charakter dieser Erscheinung zu überzeugen: Dort, wo du einen Toten suchtest, da fandest du zwei Lebende. Es geht also um den Gegensatz von Tod und Leben. Eckhart dagegen wendet die Stelle anders und er beantwortet dabei gleichzeitig die Frage, warum die Trauernde an den Engeln keinen Trost finden kann: ez ist gotes eigenschaft, daz er ein sî. Wan si dô einen suochte und $z$ wêne vant, dar umbe enmochte si niht getrAstet werden. ${ }^{16}$ Damit steht am Ende der Predigt, die vom Suchen und Finden handelt, Eckharts zentraler Gedanke vom göttlichen Unum. Bei aller Vorlagentreue im Detail wird die Predigt doch umgewertet: Es geht nicht mehr um die Verteidigung der Affekte, sondern um die Rückkehr ins Eine.

Dies ist auch die zentrale Aussage der Predigt 56, die - an sich schon sehr kurz - nur reduziert überliefert ist, weil sie vom Redaktor der berühmten Sammlung des 'Paradisus anime intelligentis' auf ihr schieres Gerüst gekürzt wurde. Trotz der Kürze lässt sich der zentrale Impetus aber noch deutlich erkennen. Eckhart will zeigen, dass die Seele Gott allein suchen soll. Er folgt auch hier seiner Vorlage genau, wenn er sagt: Diu sêle, diu got suochen sol, alle crêatûren suln ir ein pîne sîn. Ir was ein pîne, daz si die engel sach. Alsô suln der sêle, diu got suochen sol, alliu dinc sîn als ein niht. Dies ist einer der ,Kernsätze' aus Eckharts Lehre (Alle crêatûren sint ein lûter niht). ${ }^{17}$ Und dieser berühmt gewordene Satz findet sich schon in der Pseudo-Origenes-Homilie vorgeprägt: Ego enim quaero creatorem et ideo grauis est mihi ad uidendum omnis creatura. ${ }^{18}$ Ebenso lassen sich alle sechs Stücke, die Eckhart am Ende seiner Predigt kurz auflistet, und von denen er sagt, dass die Seele, die Gott finden will, sie haben muss, in seiner Vorlage finden: Dass die Dinge des Irdischen bitter sein müssen; dass die Seele

${ }^{16}$ DW II, S. 584f. Eckhart behandelt das Thema der Einheit häufiger gerade anhand des Treffens Maria Magdalenas und der Engel. Vgl. DW II, S. 86f. und 119.

${ }^{17}$ DW II, S. 589,7ff. und Anm. 4; der 'Kernsatz' DW I, S. 69,8ff.: Alle crêatûren sint ein lûter niht. Ich spriche niht, daz sie kleine sîn oder iht sîn: sie sint ein lûter niht.

18 Patera [Anm. 14], S. 442, $243^{\text {b }}$ sl. 2. Eckhart zitiert diesen Satz aus der PseudoOrigenes-Predigt sowohl im Johanneskommentar als auch im lateinischen Sermo VIII: LW III n. 706 und LW IV S. 81,4f. 
zu eng sein muss, so dass sie nicht in ihr selbst bleiben kann; dass sie nichts außer Gott suchen soll; dass sie niemand außer Gott trösten kann; dass sie alle vergänglichen Dinge übersteigen muss und dass sie außer in Gott keine Ruhe findet. Eckhart ist hier insgesamt erstaunlich wenig eigenständig (wohl weil die Pseudo-Origenes-Homilie seinen eigenen Auffassungen so genau entspricht - und weil es sich bei diesen beiden Predigten eher noch um Entwürfe als um fertig ausgeführte Texte handelt?); aber die Detailtreue, mit der er seiner Vorlage folgt, ist dann doch frappant. Bis dahin, wo er den Gegensatz von Tod und Leben neu fasst: als Vielfalt und Einheit. Nicht die Redaktion der Trauernden ist sein Thema, sondern die Einheit der suchenden Seele mit Gott.

\section{2.}

Der zweite Prediger, der hier erwähnt werden muss, ist der Franziskaner Marquard von Lindau, bezeugt ab 1373, gestorben 1392. Während Eckhart seit Langem im Zentrum des Forschungsinteresses steht, wurde Marquard lange Zeit vernachlässigt, obwohl es sich bei inm um den „produktivsten Autor des Franziskanerordens in Deutschland in der 2. Hälfte des 14. Jahrhunderts" 19 handelt und man in ihm einen „Wegbereiter 'moderner' Spiritualität" zu sehen hat, mit dem "die deutsche Predigt des 14. Jahrhunderts einen letzten Gipfel ersteigt." ${ }^{20}$

1373 ist er als Lesemeister am Studium der oberdeutschen Franziskanerprovinz in Straßburg bezeugt; nach einem Bericht aus dem 18. Jahrhundert war er auch Lektor am Würzburger Franziskanerkloster; 1377 und 1382/83 ist er als Kustos der Konstanzer Kustodie beurkundet; 1377 wird er als Minister generalis seines Ordens im Auftrag des Gegenpapstes Clemens VII. zum Magister der Theologie ernannt; er

\footnotetext{
${ }^{19}$ Bahnbrechend: Nigel F. Palmer: Marquard von Lindau, in: ${ }^{2}$ VL Bd. 6 (1987), Sp. 81-126, hier Sp. 81.

${ }^{20}$ Georg Steer: Geistliche Prosa, in: Ingeborg Glier (Hg.): Die deutsche Literatur im späten Mittelalter (1250-1370). Zweiter Teil: Reimpaargedichte, Drama, Prosa (Geschichte der deutschen Literatur von den Anfängen bis zur Gegenwart III/2), München 1987, S. 306370, hier S. $338 f$.
} 
schließt sich dennoch nicht der avignonesischen Obedienz an, sondern bleibt Urban VI. treu; von 1389 bis zu seinem Tod am 13.08.1392 ist er Provinzial der oberdeutschen Provinz.

Marquard hat Eckhart gekannt, dessen Werk in extenso benutzt und stellenweise seine Quellen und Autoritätenzitate auch über Eckhart vermittelt bekommen. ${ }^{21} \mathrm{Im}$ vorliegenden Fall ist dies anders; Marquard greift nicht auf Eckhart zurück, sondern benutzt - wie dieser - direkt die Pseudo-Origenes-Homilie über Maria Magdalena am Grab; allerdings wählt Marquard andere Stellen als Eckhart aus und fügt sie in einen völlig anderen Kontext ein, denn Marquard verfolgt einen anderen Zweck: Er predigt über Lc 10,42 (Optimam partem elegit) am Festtag der heiligen Maria Magdalena (22. Juli); wie fast immer bei Marquard, ist auch diese Lesepredigt nach dem für inn typischen Muster aufgebaut: Das Thema wird „in drei Teile (stuk) gegliedert, [...] jeder dieser Teile $[\ldots]$ in sechs Punkten abgehandelt." 22 In seiner Predigt von der $h$ ?h gelobten sant marien Magdalenen will Marquard also seinem Muster entsprechend drú stuk ausführen:

Ze dem ersten, wie die wirdig Maria Magdalena in sechs dingen $h \forall t$ daz best erwellet. Ze dem andern, wie si h?rt daz wort gottes, do si sass $z \Gamma$ sinen $f \Phi s s e n$. Ze dem dritten, wie si die drissig iar in der wústi vertraib. ${ }^{23}$

Anders als Pseudo-Origenes und Eckhart, und dabei eher in der Tradition Odos von Cluny, geht es Marquard also um ein Gesamtbild, um die Darstellung der wichtigsten Stationen des Magdalenen-Lebens. Dabei spielt die Szene am Grab natürlich eine wichtige Rolle, und hier, für diesen Teil, schließt sich Marquard dem Pseudo-Origenes-Text an. Allerdings schreibt Marquard nicht den Vorlagentext komplett ab,

${ }^{21}$ Freimut Löser: Rezeption als Revision. Marquard von Lindau und Meister Eckhart, in: Beiträge zur Geschichte der deutschen Sprache und Literatur 119 (1997), S. 425-458.

${ }^{22}$ Rüdiger Blumrich: Marquard von Lindau, Deutsche Predigten (TTG 34), Tübingen 1994, S. 47*. Im Folgenden werden Marquards Texte nach dieser Ausgabe zitiert; vgl. meine Rezension in: Beiträge zur Geschichte der deutschen Sprache und Literatur 118 (1996), S. 493-499.

${ }^{23}$ Blumrich [Anm. 21], S. 301,10-13. 
sondern verwendet nur Exzerpte daraus; und er verfolgt, wie Rüdiger Blumrich für seine Predigtweise generell festgestellt hat, ein anderes Ziel, das inn von seiner Quelle Pseudo-Origenes unterscheidet: „Höhepunkt und Ziel seiner praedicatio ist nicht die imitatio und compassio Christi, sondern eine 'Theologia mystica' im Anschluß an Dionysius. "24 Der Weg der vorliegenden Predigt führt denn auch über einen Einstieg mit Pseudo-Origenes hin zu Pseudo-Dionysius, so er beschribet des gemФtes stilli vnd vinstern $\Phi s t .^{25}$ Auch die direkten Zitate aus Pseudo-Origenes, die Marquard verwendet, zeigen, dass es inm mehr noch als Eckhart - um den Begriff der unio geht, der für die Figur der Maria Magdalena beispielhaft aufgezeigt werden soll. Eingangs schildert Marquard Maria Magdalena in engem Anschluss an PseudoOrigenes, wie sie so gar sich

kert von allen creaturen, daz si verdr?ss, in dem hailigen grab die sch=nen zieren engel an ze sehend oder mit in ze redend, ir was allain vmb iren geminten Jesum, vnd vergass ir selbes so gar, daz si nit vorht die iuden noch nit fl?h von dem grab als die zwen junger. Wan ir gaist was me bi ir geminten, denn in ir selb selben, als Origenes von ir schribet. ${ }^{26}$

Soweit also folgt Marquard Pseudo-Origenes. Aber schon im folgenden Satz interpretiert er mit Blick auf Maria Magdalenas weitere Geschichte: Diss was ain edel ker, von dem si nie gewankt vnd vff dem si belaib luterlich bis an ir ende. ${ }^{27}$ Der ker, den Marquard meint, ist die Abkehr von allem Kreatürlichen, die Abkehr sogar von den Engeln und die Hinkehr zu Gott, ein ker, der für Marquard nicht punktuelle Umkehr, sondern dauernd ist, ein ker in die Einheit.

Das zweite direkte Exzerpt Marquards aus der PseudoOrigenes-Homilie ist umfangreicher und noch aussagekräftiger: Marquard zitiert seine Vorlage direkt aus einer lateinischen Quelle, wie der Eingangssatz zeigt; er verschiebt den Blick wieder auf die gesamte Geschichte der Maria Magdalena und er wählt, im Detail recht genau

${ }^{24}$ Ebd., S. 63*f.

${ }^{25}$ Ebd., S. 308, 276ff.

${ }^{26}$ Ebd., S. 301, 26-302, 31.

${ }^{27}$ Ebd., S. 302, $31 \mathrm{f}$. 
übersetzend, exakt die Stellen aus seiner lateinischen Vorlage aus, die den für inn interessanten Aspekt der Unio betonen:

\begin{abstract}
$D \forall r$ vmb ?ch Origenes sprichet: 'Denique Josepf posuit in monumentum corpus tuum etc. Herr dinen lip leit Josepf in daz grab, mer Maria begr $\Gamma b$ iren gaist da bi dir vnd so gar verainberlich, daz man lihter hett gescheiden ir sel von ir lip, denn ir sele von dinem t?ten lip. Hier vmb do si dinen lip sГcht, da sГcht si ?ch iren gaist, vnd da si dinen lip verlor, da verlor si ?ch iren gaist. $D \forall r$ vmb was es nit wunder, daz si dich nit erkant, do du ir erschintest, wan si ir selbes gaist ber?bet was, mit dem si dich solt erkant haben.'28
\end{abstract}

Der Pseudo-Origenes-Text appelliert in der Folge an den Herrn, Maria ihren gaist zurückzugeben und verteidigt ihr Verhalten als nicht sündhaft: Ihre Liebe und ihr Schmerz werden als Entschuldigungsgründe genannt. Marquard dagegen blickt voraus auf die weitere Geschichte der Heiligen und akzentruiert den Gedanken der Einheit noch deutlicher:

Diss hiczig brinnend minn traib si in armГt, in ellend vnd verschm $\forall$ cht vnd verbrant so gar alle creatúrlich liebi in ir, daz si

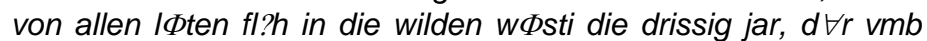
daz ir gemút alle zit vff gekeret w(re gegen iren geminten vnd mit dem allain verainb(rt wurde sunder mittel. ${ }^{29}$

Auch den Weg, ohne Mittel (!) mit Gott eins zu werden, glaubt Marquard zu kennen - und hier findet sich der stärkste Unterschied zu seiner Vorlage. Denn der Pseudo-Origenes-Text hebt am Ende die Notwendigkeit von vita activa und vita contemplativa hervor. Dem ist in einer berühmten Predigt über Maria und Martha auch Meister Eckhart

28 Ebd., S. 302, 56-303, 63. Wie genau Marquard übersetzt, zeigt ein Blick in den lateinischen Pseudo-Origenes-Text: Cum loseph posuit in monumento corpus tuum, Maria pariter ibi sepilivit spiritum suum et ita indessolubiliter iunxit et quodammodo univit eum cum corpore tuo, ut facilius posset separare animam se vivificantem a vivente corpore suo, quam spiritum (suum) te diligentem a defuncto copore tuo. Ich verzichte hier auf den Abdruck der kompletten Stelle. Vgl. Patera [Anm. 14], S. 445f.

${ }^{29}$ Blumrich [Anm. 21], S. 303, 63-67. 
gefolgt. ${ }^{30}$ Marquard hingegen entscheidet sich klar für eine der beiden Seiten:

Du waist wol, daz Mbend leben ist, so ist ?ch sch?wend leben, aber sch?wend leben ist an im selber edler, wan es sich allain mit dem obrosten grt bekúmert. Es ist ?ch im selber luter vnd $h \forall t$ me sicherhait vnd ist?ch rФwiger vnd fridlicher vnd fr=licher, wan ain sch?wender mensch $h \forall t$ nicht gegenwúrtig denn allain das ainvaltig still wesen gottes vnd st $\forall t$ in ledikait aller sorgen vnd ist frig aller bekúbernúst vnd berúrung der creaturen. ${ }^{31}$

Dies ist nun ein wichtiger neuer Trost-Gedanke: Die Freiheit von allen Sorgen, die Befreiung von jedem Kummer geht einher mit dem Entzug vom Kreatürlichen und sie liegt in der Sicherheit, Ruhe, Friedlichkeit und Freude der vita contemplativa.

In dieser Schlussfolgerung und in dieser eindeutigen Höherbewertung der vita contemplativa, die Marquard gegen seine Vorlage aus der Stelle herausarbeitet, ist inm in der weiteren Rezeptionsgeschichte der Pseudo-Origenes-Homilie freilich niemand gefolgt.

\section{III.3.}

Besonders Jan Milič von Kremsier, der als letzter Prediger zu behandeln ist, ist von anderen Auffassungen geprägt, von anderen Zielsetzungen getragen. ${ }^{32}$ Der Tscheche aus Kroměříž, dessen Predigten bisher nur in

${ }^{30}$ DW III, Predigt 86.

${ }^{31}$ Blumrich [Anm. 21], S. 304, 106-113.

${ }^{32}$ Ich übernehme die folgende Charakteristik Miličs aus meiner Darstellung in Freimut Löser: Jan Milič [Anm. 12], S. 234-237; Literatur zu Milič (in Auswahl): Jaroslav Kadlec: Milič Jan, von Kremsier, in: ${ }^{2}$ VL Bd. 6, Sp. 522-527; Villém Herold/Milan Mráz: Johann Milič von Kremsier und das hussitische revolutionäre Denken, in: Mediaevalia Philosophica Polonorum 21 (1975), S. 27-52; Howard Kaminsky: A History of the Hussite Revolution, Berkeley/Los Angeles 1967, S. 9ff.; Winfried Baumann: Die Literatur des Mittelalters in Böhmen (Veröffentlichungen des Collegium Carolinum 37), Wien 1978, S. 207ff.; Eduard Winter: Frühhumanismus. Seine Entwicklung in Böhmen und deren europäische Bedeutung für die Kirchenreformbestrebungen im 14. Jahrhundert, Berlin 1964, bes. S. 86103; Amadeo Molnár: Die eschatologische Hoffnung der böhmischen Reformation, in: 
lateinischer Überlieferung bekannt waren, muss deshalb hier besprochen werden, weil es von seiner lateinischen Fassung der Pseudo-OrigenesHomilie eben auch eine deutschsprachige Bearbeitung gibt.

Jan Milič und Marquard von Lindau sind gerade noch Zeitgenossen: Milič starb im Jahr 1374, also ein Jahr, nachdem Marquard erstmals als Lesemeister am Studium generale der oberdeutschen Franziskanerprovinz in Straßburg bezeugt ist und seine Karriere begann. Der 'Vater der tschechischen Reformation' wurde um 1320 geboren, erhielt seine Ausbildung vor der Gründung der Prager Universität in Olmütz, wirkte an verschiedenen Kanzleien, ab 1358 an der kaiserlichen in Prag, wo er unter der Leitung des Kanzlers Johann von Neumarkt seinen lateinischen Stil ausbildete. Vielleicht Johanns Befürwortung hatte er es zu danken, dass er als Kanoniker in Prag und als Administrator eines Archidiakonates eingesetzt wurde. Unter dem Eindruck der Verfallserscheinungen der Kirche und der Sittenpredigten Konrads von Waldhausen gab er seine Stelle in der kaiserlichen Kanzlei auf, verzichtete auf die kirchlichen Würden und wirkte als Bußprediger in der Pfarrkirche zum hl. Ägidius in Prag. Seine Überzeugung, dass der Antichrist in den Jahren 1365-69 auftauchen würde, führten zu Schwierigkeiten in Prag und $\mathrm{zu}$ Auseinandersetzungen mit der Amtskriche (1367 auch zu Verhaftungen und Rechtfertigungen in Rom). Nach dem Tod Konrads übernahm Jan Milič an dessen Stelle die Predigten in der Prager Theinkirche; zu diesem Zweck erlernte er auch die deutsche Sprache. Am Ende seines Lebens erneuten Angriffen ausgesetzt, reiste er nach Avignon, um sich dort zu verteidigen. Er starb dort am 29. Juni 1374 - ca. 50 Jahre nach Meister Eckhart. Bedeutsam sind seine kirchenreformatorischen Schriften und die Gebete, von denen einige auch in deutscher Sprache überliefert sind. Diese Gebete stehen häufig mit den Predigten in Verbindung, eine Verbindung, die sich früh auflöste, weil die Gebete in Gebetbüchern aufgenommen wurden. In der Edition durch Josef Klapper lässt sich der ursprüngliche Kontext der Gebete deshalb nicht mehr rekonstruieren. ${ }^{33}$ Milič trug sie nicht nur vor und nach der Predigt vor, sondern auch nach dem Vortrag des

Josef L. Hromádka (Hg.): Von der Reformation bis zum Morgen (= Od reformace k zítřiku), Leipzig 1959, S. 59-188, bes. S. $62 \mathrm{ff}$.

${ }^{33}$ Josef Klapper (Hg.): Schriften Johanns von Neumarkt Bd. 4: Gebete (Vom Mittelalter zur Reformation Bd. VI/4), Leipzig 1935, Nr. 19 und 30-32. 
Prothemas. Jan Milič entfaltete seine enorme Wirkung vor allem als Prediger. Es ist bezeugt, dass er häufig mehrmals am Tag predigte - in lateinischer, tschechischer und deutscher Sprache. Erhalten sind nur lateinische Predigttexte. Sie sind nicht ediert, und bis auf sehr wenige Ausnahmen auch nicht untersucht. Neben den Synodalpredigten und verschiedentlich erhaltenen Einzelpredigten sind hier vor allem folgende Sammlungen zu nennen:

- 'Abortivus': eine Postille mit 106 Predigten, die Milič in den Jahren 1365-66 auf Wunsch der Hörer aus den Reihen der Prager Studenten verfasste.

- 'Gracia dei', eine ebenfalls zweiteilige Postille aus den Jahren 1368-72.

- Die zwei Sammlungen der 'Sermones quadragesimales' vom Ende seiner Predigttätigkeit (also nach 1372). Man spricht auch von den 'Prothemata sermonum per quadragesimam', da nur die Prothemata ausgearbeitet wurden, während die eigentlichen Predigten (vielleicht aus Zeitmangel) in der Regel nur angedeutet sind. ${ }^{34}$

Aus der letztgenannten Sammlung stammt die Predigt und das Prothema, um das es hier geht. Ich zitiere nach dem Codex der Münchner Staatsbibliothek, clm 3097. ${ }^{35}$ „Die Predigten Miličs bestehen“, wie Jaroslav Kadlec es formuliert hat, "größtenteils aus lateinischen Bibelzitaten und aus Zitaten aus der patristischen Literatur. Das ermöglichte es dem Prediger beim Vortrag in der Volkssprache, volle Ausdrucksfreiheit zu wahren." ${ }^{36}$ Kadlec spricht also von lateinischen Entwürfen und Materialsammlungen, die dann beim Vortrag in der Volkssprache ad hoc ausgearbeitet wurden. Ich will auf das bekannte und virulente Problem Schriftlichkeit/Mündlichkeit in der Predigt hier nicht eingehen. Denn Miličs mündliche Rede ist für mich nicht rekonstruierbar;

${ }^{34}$ Kadlec [Anm. 31], Sp. 526.

35 Initien bei Johannes Baptist Schneyer: Repertorium der lateinischen Sermones des Mittelalters für die Zeit von 1150-1350, Bd. 3 (Beiträge zur Geschichte der Philosophie und Theologie des Mittelalters XLIII/3), Münster 1971, S. 583-588.

${ }^{36}$ Kadlec [Anm. 31], Sp. 526. 
sein Verhältnis zu seiner Quelle hingegen lässt sich sehr genau bestimmen und da lässt sich feststellen: Milič hält sich bemerkenswert treu an den Pseudo-Origenes-Text. Er selbst legt seine Quelle schon zu Beginn seiner Predigt offen: Origines in homelia dicit exponens evangelium sic de praesenti solemnitate locuturus... ${ }^{37}$ Man sieht, dass Milič von den beiden Versionen diejenige benutzte, deren Prolog den Bezug zum Tag der heiligen Maria Magdalena schafft. ${ }^{38}$ Feststellbar ist zunächst eine Genauigkeit im Detail: Miličs Text folgt der Vorlage noch sehr viel stärker als Eckhart und Marquard von Lindau. Im Grunde wird sie einfach nur wiedergegeben. Dies liegt weniger daran, dass es sich bei Miličs Predigt um einen lateinischen Sermon, bei Eckhart und Marquard um volkssprachliche Predigten handelt. Milič ging es offensichtlich darum, mit der Wiedergabe der Pseudo-Origenes-Homilie zunächst einmal nur den Stoff für die eigene Predigt zur Verfügung zu haben; einen Stoff, der inm entgegen kam und der für seine Zwecke verwendbar war. Einzelne Kürzungen straffen den Text und machen ihn leichter zugänglich. Milič zielt stärker als seine Vorlage auf die ehemalige Sünderin Maria Magdalena und betont eigens, dass der Herr ihr die Sünden vergeben hatte. ${ }^{39}$ Die Verteidigung zielt somit weniger auf eine Legitimation des Affekts und der Trauer, als vielmehr darauf zu betonen, dass Maria seit der Zeit, als der Herr ihr die Sünden vergab, keine Sünden mehr begangen hat. Der Apell des Predigers richtet sich an den Herrn und fragt, warum er sich einer Sünderin entzieht, deren einzige denkbare 'Sünde' darin bestanden haben könnte, sich ihm ganz zuzuwenden. ${ }^{40}$ Die Tatsache, dass der Herr sich entzieht, wird im Pseudo-Origenes-Text so kommentiert: Pascha enim ab isto deffuncto non contaminatur, sed renovatur. Mortuus iste non polluit mundos, sed mundat immundos. ${ }^{41}$ Milič ergänzt ausdrücklich: Christi Kreuzestod ist um der Sünder willen vollbracht worden. ${ }^{42}$ Milič verstärkt also eine

37 Schneyer [Anm. 34], S. 586 Nr. 102 T 28/5.

${ }^{38}$ Auch das Explizit entspricht exakt der lateinischen Vorlage: vidi Dominum et haec dixit mihi, cui est honor et gloria... (vgl. ebd.).

${ }^{39}$ Vgl. Melk, Stiftsbibliothek, cod. 1865 (olim 586 / antea L5), f. $172^{\text {va }}$.

${ }^{40}$ Ebd., f. $172^{\text {va-b. }}$.

${ }^{41}$ Louis Bourgain, La Chaire Française au XII Siécle d'áprés les Manuscrits, Paris 1879 (Nachdruck Genf 1973), S. 379.

${ }^{2}$ Melk, Stiftsbibliothek, cod. 1865 , f. 173 vb. 
Tendenz, die bei Pseudo-Origenes schon angelegt ist. Am Ende des Textes werden die Zuhörer aufgefordert, von einer Sünderin zu lernen, der ihre Sünden vergeben wurden. Weshalb diese Tendenz des Textes und diese Interpretation Jan Milič besonders entgegenkam, ist ohne weiteres einsichtig: Seine Beschäftigung mit der Pseudo-OrigenesHomilie datiert aus der Zeit um 1372. Dies war das Jahr in dem Milič, so Kadlec, „den größten Erfolg erzielte“, als er eine große Zahl Prager Prostituierter zu einem Leben in freier religiöser Gemeinschaft bekehrte. Oder, um es mit Amadeo Molnár zu formulieren:

„He gave the name of Jerusalem to a shelter for fallen woman, built with great effort. He chose a name full of significance. Miličs house of prayer and work, built in the very centre of the capital of the Czech kingdom, on today`s Konviktská Street, became the symbol of the Lord`s new people." 43

Dass die Kirche dieser Institution der heiligen Maria Magdalena geweiht war, wird niemanden verwundern. Milič steht zu dieser Zeit im Zentrum einer Bewegung, die sich den Sünderinnen zuwendet, und ihnen im Zeichen der Heiligen den Weg weist. ${ }^{44}$ In diesen Kontext gehört Miličs Rezeption des Pseudo-Origenes-Textes. Es ist deshalb nicht verwunderlich, dass die "mystischen“ Aspekte des Textes zugunsten einer aszetisch orientierten Spiritualität zurückgedrängt werden. Im Prothema, das Milič eigenständig ausarbeitet und dem Origenes-Text voranstellt, nehmen diese ethisch, moralisch und didaktisch orientierten Akzente eine sehr konkrete Richtung; dazu wird ein Thema aufgegriffen, das in der Vorlage nur kurz anklingt: mulier iste que valde mane vigilat ad te. ${ }^{45}$ Valde mane wird zum Leitbegriff des Prothemas: Der Text der Bibel sei gegen die Langschläfer gerichtet, die die Predigt und den Gottesdienst versäumen. Man solle nicht schlafen wie die Grabeswächter oder zögerlich kommen wie die Jünger, sondern früh schon wie Maria Magdalena den Herrn mit andächtigem Gebet suchen. Dies ist natürlich literaliter und spiritualiter zu verstehen. Milič untersucht

${ }^{43}$ Milan Opočenský/Jana Opočenská (Hgg.): The Message fort the last days. Three Essays from the Year 1367. Milič of Kroměříž. Introduction by Amadeo Molnár, S. 7-17, hier S. 16. ${ }^{44}$ Literatur dazu bei Löser, Jan Milič [Anm. 12], S. 237, Anm. 47.

${ }^{45}$ Bourgain [Anm. 40], S. 376. 
mithin weniger die Trauer (und schon gar nicht die Affektladung des Trauergefühls), sondern die Hoffnung. Sein Appell richtet sich gegen den (Sünden-)Schlaf.

\section{IV.}

Damit stehen wir für die deutsche Predigt des Mittelalters am Ende der Anverwandlung der Pseudo-Origenes-Homilie, soweit ich bisher sehe. ${ }^{46}$ Ich bin allerdings sicher, dass aus dem ungedruckten Material der Handschriften noch weitere, womöglich zahlreiche Vergleichstexte zutage treten werden. Und mit den Predigten ist das Thema längst nicht erschöpft. Denn die Pseudo-Origenes-Homilie wirkte auch in anderer Form: Es gibt, soweit sie mir bekannt geworden sind, fünf ProsaÜbersetzungen allein ins Deutsche: ${ }^{47}$ Ich habe sie an anderer Stelle

${ }^{46}$ Der bekannte Prediger aus dem Kreis der Devotio moderna, Johannes Brinckerinck (vgl. ${ }^{2} \mathrm{VL} \mathrm{Bd} .1$, Sp. 1037f.) behandelt in der ersten seiner Collacien zwar das Thema Maria Magdalena sochte den here vroe in der morghenstont, rekurriert dabei aber an keiner Stelle auf die Pseudo-Origenes-Homilie. Vgl. W. Moll: Acht Collati*n van Johannes Brinckerinck, in: Kerkhistorisch Archief 4 (1866), S. 97-168, hier S. 111-121. Zum Niederländischen: Rossano [Anm. 1].

47 Rossano [Anm. 1] behandelt zwar „die deutschen und niederländischen [!] Bearbeitungen“, übersieht aber offenbar gerade die Texte, auf die schon sehr früh hingewiesen worden war: Kurt Ruh: Bonaventura deutsch, Bern 1956, S. 224 weist - im Rahmen seiner Untersuchung der niederländischen Übertragung Legenda S. Francisci auch auf eine niederländische Prosabearbeitung hin: Den Haag, Kgl. Bibl. 75 G 13 (Nr. 780) [Hg. 9], f. $205^{r}-212^{v}$ aus dem Jahr 1443 von der Hand der Schwester Barbele Sermertens; weiter nennt er dort Leiden, Letterk. 312 f. 159r-183 (vgl. dazu auch John P. McCall: Chaucer and the Pseudo Origen De Maria Magdalena: A Preliminary Study, in: Speculum 46 (1971), 491-509; hier S. 508); Wolfenbüttel, Helmst. 1268, f. 69'-96v . Die Bedeutung der niederländischen Übersetzungen ist deshalb eminent, weil auch Hadewijch die Pseudo-Origenes-Homilie gekannt und verwendet hat (Mengeldicht III, 50-68). Gründlich untersucht und dargestellt hat dies Frank Willaert, Hadewijch en Maria Magdalena, in: Miscellanea Neerlandica (Festschrift für Jan Deschamps zum 70. Geburtstag), hg. von Elly Cockx-Indestege und Frans Hendrickx, Bd. II, Leuven 1987, S. 57-69 (hier S. 64, Anm. 33 auch zu weiteren niederländischen Hss.). Die Übersetzung der Pseudo-Origenes-Homilie durch den Kartäuser Nikolaus Kempf ( ${ }^{2} \mathrm{VL}$ Bd. 4, Sp. 1117-1124, hier Sp. 1122) ist wohl mit der Bibliothek der Kartause Gaming verloren gegangen. Vgl. dazu A. Hörmer: Der Kartäuser Nikolaus Kempf als Seelenführer, Diss. Masch, Wien 1959, S. 8f., Nr. 36 und besonders Nigel F. Palmer: Ein Handschriftenfund zum 
etwas ausführlicher behandelt ${ }^{48}$ und beschränke mich hier auf einige Grundsatzpositionen und Ergänzungen zu den hier relevanten Themen: ${ }^{49}$

\section{IV.1.}

'Die Mittelfränkische Übertragung' der Donaueschinger Handschrift cod. B VI 2 (16. Jahrhundert; vorlagentreu) ist hier nicht ergiebig.

\section{IV.2.}

Bei der frühesten deutschen Bearbeitung handelt es sich um ein eigenes, Maria Magdalena (und ihren 'Geschwistern' Martha und Lazarus) gewidmetes Bändchen, das zusammen mit einer ganzen Gruppe ähnlicher libelli (Johannes Baptista, Johannes Evangelista, Klara) wahrscheinlich noch im 14. Jahrhundert in Nürnberg angelegt oder zusammengestellt wurde. ${ }^{50}$ Der Text, der Werner Williams-Krapp zufolge noch Reste der alemannischen Sprache aufweist, bietet eine Zusammenschau des Lebens der Heiligen. Dass dabei auch die Szene am Grab eine Rolle spielt, versteht sich. Ich konzentriere mich im Folgenden darauf.

Für die Gesamtkonzeption sei nur der Prolog erwähnt, der die Zielrichtung deutlich macht: Der Name 'Maria' signalisiert Reue, Contemplatio und himmlische Ehre; dem werden die Begriffe Bitterkeit, Süßigkeit und Erleuchtung zugeordnet. 'Magdalena' bezeichnet die Schuld und die Buße zu Füßen des Herrn; dass Magdalena sich wol gewarnt hat, meint ihre Demut; dass sie nach ihrem Leben groz und

Übersetzungswerk Heinrich Hallers und die Bibliothek des Grafen Karl Mohr, in: Zeitschrift für Deutsches Altertum 102 (1973), S. 49-66, hier S. 53 und 58.

${ }^{48}$ Löser, Jan Milič [Anm. 12], S. 237-244.

49 Text-Abdrucke großenteils bei Madeleine Boxler: "ich bin ein predigerin und appostlorin". Die deutschen Maria Magdalena-Legenden des Mittelalters (1300-1550). Untersuchungen und Texte (Deutsche Literatur von den Anfängen bis $1700 \mathrm{Bd} .22$ ), Bern u. a. 1996, Nr. 8.31, S. 524-536; vgl. die Rezension durch Werner Williams-Krapp, in: Zeitschrift für Germanistik NF 2 (1998), S. 443ff.; vgl zur Kritik vor allem Löser, Jan Milič [Anm. 12], S. $237 f$.

${ }^{50}$ Vgl. Werner Williams-Krapp: 'Maria Magdalena', in: ${ }^{2}$ VL Bd. 5, Sp. 1258-1264; hier Sp. 1261. 
wirdig wurde, ist durch die übergroße göttliche Gnade bewirkt worden. Damit sind alle zentralen Begriffe genannt, auf die hin auch das Geschehen am Grab gedeutet wird. Die Szene wird mit einem Evangelienzitat eingeleitet, sie ist ganz nach der Pseudo-OrigenesHomilie gestaltet und reicht (mit folio $50^{\mathrm{v}}-81^{\mathrm{r}}$ ) über ca. 30 Blätter des Codex. Schon der Textbeginn ist aufschlussreich. Er führt nämlich aus, dass Johannes der IMter tavgen schawer der hiligen trivaltikeit der daz wirdig lob diser froven in seinem ewangelio vz genMmenlich wol erh $=h t$ hat [...] ovch von dirre materie vorderlich geschriben [hat] dann di andern Evangelisten. ${ }^{51}$ Damit wird der Bezug zu dem Johannes-libellus derselben Serie gesucht. ${ }^{52}$ Die Auslegung aber folgt der PseudoOrigenes-Homilie und schon der Beginn der Stelle hebt dies explizit hervor: daz selb ewangelium bedevtet der hohe lerer Origenes mit einer sMzen Omely, der ein teil tr=stlich hie zesagen ist. ${ }^{53}$ Die Angabe, hier werde ein teil der Homilie wiedergegeben, ist nur insofern zutreffend, als einige kleinere Kürzungen vorgenommen werden. Sie betreffen vor allem die Klagereden Maria Magdalenas, die nicht in extenso vorgetragen, sondern zusammengefasst werden: Owi manig andehtigen rvf vnd gebet sy tet. ${ }^{54}$ Ansonsten folgt der Text seiner Vorlage relativ treu, versucht aber den vielfältigen Perspektivenwechsel der Vorlage zu erklären und zu kommentieren; z. B. so: Nv redet der lerer Origenes mit der person diser got iamerigen marien vnd spricht also. ${ }^{55}$ Auch die Szene der Homilie, in der das Ich der Predigt sich an Christus wendet, um Maria Magdalena zu verteidigen, wird leicht verändert: $O d v$ sMzzer vnd $d v$ milter meyster christe, ich dein diener Origenes entar hie niht entschvldigen dein minneriche jMngerin. ${ }^{56}$ Diese Notwendigkeit der 'Entschuldigung' Maria Magdalenas arbeitet der Nürnberger libellus noch

${ }^{51}$ Ich zitiere - unter Vorbehalt und mangels eines Mikrofilmes - nach dem Abdruck bei Boxler [Anm. 48] (hier S. 267, 53'), löse jedoch die Kürzel auf und führe eine Zeichensetzung ein.

${ }^{52}$ Auch die nun folgende Bearbeitung der Pseudo-Origenes-Homilie betont an den Stellen, an denen das Evangelium selbst zu Wort kommt, über die Vorlage hinaus immer wieder, der Evangelienbericht stamme vom hymlisch adelar Johannes oder vom minnerich Johannes (ebd., S. 247, $74^{r}$ und $271,67^{\text {r }}$; vgl. S. 275, $77^{\text {r) }}$.

${ }^{53}$ Ebd., S. 267, 53r.

${ }^{54}$ Ebd., S. 270, 63r

${ }^{55}$ Ebd., S. 270, 61 ${ }^{\mathrm{v}}-62^{\mathrm{r}}$.

${ }^{56}$ Ebd., S. 272, 69v. 
stärker als seine Vorlage bemerkenswert klar heraus. Als sie zum Grab kommt, sucht sie in ihrer Verzweiflung Trost: wann der si in allen irem leide sMzzelich getrost het den het sy gegenwertiklich verloren ${ }^{57}$ und sie glaubt, im Grab den Leib Christi zu finden, der sie trösten soll: Nv waz ein engel von hymel kvmen vnd het den grozzen stein von dem leren grab gewelczet; daz waz ir tr=stlich, vmb daz sy des paz zv dem lichnam irs liben herren kvmen $m=h t .{ }^{58}$ Trost über den Tod beim Toten zu suchen - das ist in christlichem Sinn ein fulminanter Irrtum, denn der wahre Trost läge für Maria Magdalena (und liegt für die Christenheit) im Gedenken an die Verkündigung der Auferstehung und der Überwindung des Todes durch Christus selbst: wann so het si wol gewizzet, daz si in niht mer als einen toten klagen noch svchen $s=1$ in dem grab, svnder si het sich ser gefrewet, vmb daz er warer got vnd mensch waz. ${ }^{59}$ Hindernis dieses Vertrauens in die Auferstehung ist die Verzweiflung über den Tod: da von ward ir vernMnftig bescheidenheit also ser vberladen mit trvrigen inpildvnge, daz si ein teil irrend oder zwivelnd ward vf dem wege der warheit cristenliches gelavben an der vrstend vnsers herren. ${ }^{60}$ Und hier nun - an dem Punkt, wo das Leid in Glaubensirrtum umschlagen kann - greift der 'Entschuldigungsgedanke'; denn der zwivel ist nicht Ausfluss der Verzweiflung oder des Zweifels an Gott, sondern der großen Liebe zu Gott:

ich dein diener Origenes entar hie niht entschvldigen dein minneriche jMngerin. Jch mag sy Mrilich niht beschirmen von der irresal, di sy gehabt hat in der warheit deiner vrstend. Aber ich mag vnd will eygenlich sprechen, das dise irresal in irem herzen von keiner hertikeit noch von keinem vngelovben gewurczelt ist, svnder alein von grozzer minne vnd von tiefem leide, das ir verstantnMsse so ser vberladen het, vmb daz sy dich, iren geminten, verloren het. Da von solt $d v$, barmherziger vnd gerehter rihter, vbertragen di

${ }^{57}$ Ebd., S. 268, 57

${ }^{58}$ Ebd., S. 268, 55v

${ }^{59}$ Ebd., S. 269, $59^{\text {r }}$

${ }^{60}$ Ebd., S. 268, 58'. 
irresal diser betrMbten frowen vnd solt an sehen di minne prinnenden begirde di sy zv dir hat. ${ }^{61}$

Der Text ist darüber hinaus stark beeinflusst von der Diktion und der Denkweise der deutschen Mystik eckhartscher Prägung, etwa, wenn er die Vorlage verändernd - erklärt, die Tatsache, dass Maria nicht am Trost der Engel genug habe, sei nicht nur darauf zurückzuführen, dass sie den Herrn der Engel allein sucht (soweit noch Origenes), sondern auch:

Di sch=n der engel mag meinem herzen kein genMg tun, wann ich svch den, der mich mit adelicher fr $\square$ heit nach im selber gepildet hat vnd der sich von grozzer minne miner br=den natvr gelichet hat. ${ }^{62}$

Derartige Erweiterungen sind nicht selten und sie nehmen gegen Schluss der Homilie spürbar zu. Das Verfahren ist kompilatorisch; man zieht neben Origenes weitere Verfasser heran, die der eigenen Interpretation des Textes entsprechen:

Ovch von der grozzen wirdikeit (man vergleiche den Prolog), do mite er si fMrtreffenlich erh=ht hat in der zit siner vrstend, schribent uns di seligen meister der hiligen cristenheit Ambrosius, Avgustinus vnd Gregorius gar svnder tr=stlich. ${ }^{63}$

Die Trauer Magdalenas wird zu ihrer Würde. Nicht ihr Trost steht im Interessenszentrum des neuen Textes, sondern der Trost des Publikums, zu dem ihre Fallbetrachtung wird. Besonders im Anschluss an die Homilie 25 des Gregorius geht es nun um die grozze wirdikeit $t^{64}$ Maria Magdalenas, die der grvndlos[en] miltikeit des Herrn zu verdanken sei, der di trMrigen klag diser edelin frowen so gar gnediklich verwandelt hat in gancz freude. ${ }^{65}$ Hier wird also einerseits bewusst der Anschluss an

${ }^{61}$ Ebd., S. 272, 69v-70r.

62 Ebd., S. 269, 61 r. Vgl. beispielsweise Alois M. Haas: Meister Eckharts geistliches Predigtprogramm, in: Ders.: Geistliches Mittelalter, Freiburg/Schweiz 1984, S. 317-337.

63 Ebd., S. 275, $78^{r-v}$.

${ }^{64}$ Ebd., S. 275, $78^{r}$.

${ }^{65}$ Ebd., S. 275, 78'. 
die Gnadentheologie gesucht. Andererseits wird das Bild Maria Magdalenas als das eines Anti-Typus zur Sünderin Eva - auch im Anschluss an Gregorius - formuliert. ${ }^{66}$ Maria Magdalena, den Leserinnen als Ivter ebenpilde vorgestellt, ist exemplarisch, indem sie got gehorsam ist; sie ist eine $v z$ erweltev zwelfpotin und hat allen frowen mit hohen garden [?=Gnaden] eramt [erarnt?=verdient] ein ewig lob grozer wirdikeit. ${ }^{67}$ Der aus heutiger gender-orientierter Sicht höchst interessante Text geht inhaltlich durch dieses 'Lob der Frauen' am Ende über die Vorlage der Pseudo-Origenes-Homilie weit hinaus und ist in eine Reihe zu stellen mit Christines de Pizan Rekurs auf Maria Magdalena und deren Verteidigung der weiblichen Eigenschaften: Christine beruft sich auf Maria Magdalenas Tränen, ihre Zeugenschaft und ihre große Liebe, wenn sie „närrischen Predigern“ gegenüber klarstellt, das angeblich so „weibisches Flennen und Schwätzen" in Wahrheit Eigenschaften seien, die Gott an den Frauen schätze: „Oh, wie viele Gnadenbeweise gewährte Gott Frauen um ihrer Tränen willen! Die Tränen der Maria Magdalena verachtete er keineswegs, sondern nahm sie an und verzieh ihr dafür ihre Sünden, so dass sie nun dank jener Tränen im Himmel wohnt." Die Trauernde muss nicht getröstet, die Tränen müssen nicht getrocknet werden; Trauer und Tränen sind der Weg zum Himmel. Dass manche Männer verbreiten, der Auferstandene habe sich Maria Magdalena nur deshalb als erster offenbart, weil er wusste, dass sie nicht würde schweigen können, sei eine Torheit und spotte Gottes. Die gewaltige weibliche Liebe habe Gott selbst in das Herz der Maria Magdalena gesenkt und gutgeheißen. ${ }^{68}$ Schließlich mache diese Liebe die Frau zur Gottes-Verkünderin. Auch im Nürnberger Maria-Magdalenalibellus ist die Verkündigung der Auferstehung ihr ausdrücklicher Verdienst:

Aber daz Maria got selber gehorsam waz vnd seinen jvngern kvndet di waren ere siner herrenlichen vrstend, an dem teil

${ }^{66}$ Ebd., S. 276, 80'.

${ }^{67}$ Ebd., S. 276, $79^{v}$ und $81^{r}$.

${ }^{68}$ Christine de Pizan: Das Buch von der Stadt der Frauen. Aus dem Mittelfranzösischen übersetzt, mit einem Kommentar und einer Einleitung versehen von Margarete Zimmermann, Berlin 1986, S. 59ff. und 251. 
fvrtriffet si alle zwelfpoten vnd ist also gr=zlich gewirdigt worden, daz sy di hilig cristenheit gar sicherlich nennet ein vz erweltev zwelfpotin, di vns daz war leben Christi gepredigt hat ${ }^{69}$.

Diese öffentliche Verkündigung der Auferstehung bewirkt die hohe Ehre Maria Magdalenas, sie ist ihr wahrer Trost im Leid und sie macht diesen Trost zu einem allgemeinen Trost:

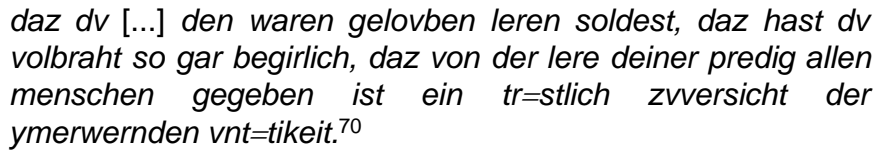
volbraht so gar begirlich, daz von der lere deiner predig allen menschen gegeben ist ein tr=stlich zvversicht der ymerwernden vnt=tikeit. ${ }^{70}$

\section{IV.3-5.}

Die drei noch zu behandelnden Übersetzungen der Pseudo-OrigenesHomilie stellen diese nicht in einen größeren Legenden-Kontext, sondern behandeln sie jeweils für sich (freilich in je eigenen größeren Kontexten). Alle drei stammen sie aus den 50er- und 60er-Jahren des 15. Jahrhunderts. Alle drei führen sie in die Kontexte der Klosterreform: der Kartäuser Heinrich Haller, die Zisterzienserin Regula, der benediktinische Laienbruder Lienhart Peuger.

\section{IV.3.}

Heinrich Haller wirkte in der Kartause Allerengelberg im Südtiroler Schnals.

Er ist dort in den Jahren 1455, 1458 in Urkunden und nach 1471 in einer Handschrift genannt. Bei seinem Übersetzungswerk, das Erika Bauer eingehend untersucht hat, handelt es sich im Wesentlichen um sechs datierte Autographe aus der Zeit von 1464 bis 1471. Bauer gliedert Hallers Werk in drei Gruppen: Belehrungen und Betrachtungen

${ }^{69}$ Boxler [Anm. 48], S. 276, 79v.

${ }^{70}$ Ebd., S. 276, f. $80^{v}$. 
(beispielsweise aus den 'Vitaspatrum'), Erbauungsschriften (wie die von Nigel Palmer behandelte 'Visio Tnugdali' oder die 'Hieronymus-Briefe') und Predigten. In diese letzte Gruppe gehört auch die Übersetzung des Pseudo-Origenes-Textes. Hallers Werk ist so umfangreich, dass es hier nicht im Einzelnen aufgezählt werden kann. ${ }^{71}$ Unter den Handschriften Hallers unterscheidet Bauer Konzepte, Originale und Abschriften. Der Pseudo-Origenes-Text findet sich im Codex 1065 des Tiroler Landesmuseums Ferdinandeum in Innsbruck, ${ }^{72}$ nach Bauer eine Handschrift mit Übersetzungskonzepten, die noch vor dem ersten datierten Kodex von 1464 einzuordnen ist.

Haller selbst spricht davon, seine Übersetzungen seien nach dem text vnd etwen nach dem sinn verfertigt. „In der Regel“, so konstatiert Bauer, „hat er also die lateinische Vorlage wörtlich übertragen, und nur manchmal hat er frei übersetzt." Dennoch habe er der eigenen Zielsetzung folgend „das Lateinische pracht czue ainer schlechten gemainen teücz, die man wol versten mag."73 Wie schon die Textauswahl Hallers beweist, geht es immer um praktische pastoraltheologische Anliegen im Blick auf ein konkret gedachtes Publikum. Haller zielt auf alle, die das puech lesen oder hören lesen und sich dadurch pessern. ${ }^{74}$ Die Origenes-Homilie kann den AllgemeinBefund unterstreichen und etwas erweitern: Sie ist bemerkenswert

${ }^{71}$ Vgl. Erika Bauer: Haller, Heinrich, in: ${ }^{2}$ VL Bd. 3, Sp. 415-418 mit weiterer Literatur; dazu dies.: Zisterzienserpredigten in der Übertragung des Heinrich Haller (Kleine deutsche Prosadenkmäler des Mittelalters 7), München 1969; Dies.: Heinrich Hallers Übersetzungen im 'gemeinen Deutsch', Göppingen 1972; Dies.: Heinrich Hallers Übersetzung der Imitatio Christi, Salzburg 1982; Dies.: Wortwahl und Wortvarianz in Heinrich Hallers 'Hieronymus', Heidelberg 1984; Dies.: Heinrich Hallers Übersetzung der 'Hieronymus-Briefe', Heidelberg 1984; Dies.: Heinrich Hallers Bibelzitate, Heidelberg 1991.

${ }^{72}$ Dieser Text dort S. 284-307, gemeinsam mit den 'Hieronymus-Briefen' (S. 1-280). Boxler [Anm. 48] ist Nigel Palmers Hinweis auf eine spätere Handschrift entgangen: Innsbruck, Universitätsbibliothek 979 (nach dem Jahr 1473) versammelt in ihrem ersten Teil eine Reihe von Übersetzungen Hallers, darunter f. $81^{\mathrm{vb}}-90^{\mathrm{va}}$ auch die Pseudo-Origenes-Homilie. Vgl. Nigel F. Palmer: Visio Tnugdali, The German and Dutch Translations and their circulations in the Later Middle Ages (MTK 76), München/Zürich 1982, S. 346-349; zur Handschrift: Ders.: Ein Handschriftenfund [Anm. 46].

${ }_{73}$ Bauer: Heinrich Haller`s Übersetzungen [Anm. 70], S. 30; vgl. besonders Palmer [Anm. 71], S. 134f.

${ }^{74}$ Bauer: Haller, [Anm. 70], Sp. 416; vgl. dies.: Zisterzienserpredigten [Anm. 70], S. 20. 
vorlagentreu ins Deutsche gebracht. ${ }^{75}$ Minimale, aber sehr interessante Zusätze versuchen, Textaussagen $z u$ verdeutlichen und die Innenperspektive Magdalenas hervorzuheben, etwa, wenn der lateinische Text sagt: Cum Maria sic doleret et sic fleret, et cum hec dixisset. ${ }^{76}$ Haller gibt dies so wieder: vnd da Maria also weinet vnd also klaget vnd daz also het geret in de $m$ hertze $n .{ }^{77}$ Der äußere Monolog wird zu einem inneren. Wo bei den poetischen Bearbeitungen, wie in Teil I beschrieben, Gesten der Trauer in den Vordergrund treten und nach Öffentlichkeit streben, erfolgt hier der Rückzug auf das Selbst und die Konkretisierung der Innenperspektive. Leid und Verzweiflung der Maria Magdalena werden zudem explizit durch eine deutlichere Charakterisierung der 'Heldin' ins Wort gebracht, etwa wenn es in Erweiterung des lateinischen Textes heißt: Si waz gantz arm vnd gantz pew*gt mit den zähern. ${ }^{78}$ Diesem Leid und den Tränen - das heben alle Bearbeiter des Pseudo-Origenes hervor - steht die Tapferkeit Maria Magdalenas gegenüber. Haller ist dabei aber der einzige, der hier so weit geht, von der mandleichheit [Männlichkeit] des weibes zu sprechen. ${ }^{79}$ Die Standhaftigkiet der 'Trauer-Helden' hilft ihr die Trauer zu überwinden und macht sie im Wortsinn 'männlich'. Den Schluss-Appell des Textes, der sich im Original einheitlich an das Publikum richtet, spaltet Haller auf, indem er den ersten Teil auf sich selbst, erst den zweiten auf die Zuhörer/Leser bezieht:

darum wil ic $h$ nachvolgen der pegird des weiwes, daz ich auch müg kömen nach der parmh*rtzigkeit zu irem *ntt [...] Tu solt I*rn, tu sundiger mensch [...] Tu solt I*rn zu peweinen [...] Tu solt I*rn

\footnotetext{
${ }^{75}$ Vergleichbar ist auch Nigel Palmers Urteil über Hallers Übersetzung der 'Visio Tnugdali' [Anm. 71]: "It is only occasionally that a modification to the text represents a new interpretation, and it seems that the author has endeavoured to remain faithful to the sense of the original throughout." Vgl. Palmer, Visio [Anm. 71], S. 136.

${ }^{76}$ Bourgain [Anm. 40], S. 380.

77 Zitiert nach dem Abdruck bei Boxler [Anm. 48], S. 518, 296.

${ }^{78}$ Ebd., S. 513, 286; vgl. S. 517, 294: we mir vngelückhaftigen.

79 Ebd., S. 521, 302. Hier lässt sich Christi Verheißung an Maria Magdalena aus dem Thomas-Evangelium vergleichen: „Seht ich werde sie ziehen, um sie männlich zu machen." (Vgl. Ingrid Maisch: Maria Magdalena. Zwischen Verachtung und Verehrung. Das Bild einer Frau im Spiegel der Jahrhunderte, Freiburg/Basel/Wien 1996, S. 36).
} 
von maria iesum lieb zuhaben [...] Tu solt I*rn von maria vnd auch suchen iesum. ${ }^{80}$

Unabhängig davon, welches Publikum Haller für seine Übersetzung im Auge hat, sagt er in der kurzen Einleitung: Daz ist daz ewangeli sand iohansen [...] vnd die nachfollgung daz ist die laienpredig origenis. ${ }^{81}$ Haller hat also die Laienbrüder seiner Kartause Schnals im Auge, wenn er am Ende der Predigt so eindringlich zum Lernen aus Magdalenas Vorbild aufruft.

\section{IV.4.}

Die Übersetzung der Pseudo-Origenes-Homilie durch die Lichtenthaler Zisterzienserin Regula wurde von dieser in deren 'Buch von den heiligen Mägden und Frauen' integriert. ${ }^{82}$

Im Lichtenthaler Nekrolog ${ }^{83}$ wird für den 20. Mai 1478 vermerkt: obiit Margaretha, dicta Regula monialis. Regula ist demnach ein Beiname, der vielleicht auch als Anspielung auf die Tätigkeit der unermüdlichen Schreibmeisterin zu verstehen ist, die im Zuge der Reformierung Lichtenthals mit der Literaturversorgung des Konvents betraut war.

${ }^{80}$ Ebd., S. 522, 304 und 305.

${ }^{81}$ Ebd., S. 514, 284.

82 Vgl. zum Folgenden: Löser, Jan Milič [Anm. 12], S. 241f.; Boxler [Anm. 48], S. 537-544; Konrad Kunze: Studien zur Legende der heiligen Maria Aegyptiaca im deutschen Sprachgebiet (Philologische Studien und Quellen 49), Berlin 1969, S. 101ff.; Ders.: Die Legende der heiligen Maria Aegyptiaca (Texte des späten Mittelalters und der frühen Neuzeit 28), Berlin 1978, S. 82-87; Ders.: Alemannische Legendare (I), in: Alemannisches Jahrbuch 1971/72, S. 20-45, hier S. 29-38; Ders. (Hg.): Die Elsässische 'Legenda aurea', Bd. II: Das Sondergut, (TTG 10), Tübingen 1983, S. XXIII-XXXII; Werner Williams-Krapp: Die deutschen und niederländischen Legendare des Mittelalters. Studien zu ihrer Überlieferungs-, Text- und Wirkungsgeschichte, Tübingen 1986, Register, bes. S. 50ff. und 351f.; Ders./ Ulla Williams (Hgg.): Die Elsässische 'Legenda aurea', Tübingen 1980, S. LVILVIII; Gerhard Stamm: Regula, Lichtenthaler Schreibmeisterin, in: ${ }^{2}$ VL Bd. 7, Sp. 11311134; Ders./Felix Heinzer: Die Handschriften der Badischen Landesbibliothek in Karlsruhe, Bd. XI: Die Handschriften von Lichtenthal, Wiesbaden 1987, S. 40-43; M. Pia Schindele: Zum 500. Todestag der Sr. Regula, Erbe und Auftrag, in: Benediktinische Monatsschrift 54 (1978), S. 234-239; Edith Feistner: Historische Typologie der deutschen Heiligenlegende des Mittelalters von der Mitte des 12. Jh.s bis zur Reformation (Wissensliteratur im Mittelalter 20), Wiesbaden 1995, Kapitel 2.1.2.3.

${ }^{83}$ Zum Folgenden: Stamm, ${ }^{2}$ VL Bd. 7 [Anm. 81], Sp. $1131 \mathrm{ff}$. 
Regula entfaltete im 3. Viertel des 15. Jahrhunderts eine rege Tätigkeit als Kompilatorin und Übersetzerin. Mit Sicherheit kann man ihre Hand in etwa zehn Handschriften identifizieren, die sie ganz (oder mindestens teilweise) schrieb.

Ihre Übersetzungen verfertigte sie ihrer eigenen Aussage zufolge durch mynne und liebe der ungelerten, die das latin nit verstond und darumb manigmol vertrosz hant vil czu lesende. ${ }^{84}$ Dazu dienen ihr nit kluge geczierte wort, die die oren füllent, sunder slecht und einfaltig also sie zu luterer andacht und innikeit des hertzen wisent. ${ }^{85} \mathrm{Zu}$ den von ihr bearbeiteten Werken zählen Liturgica, Ascetica, theologischdogmatische und exegetische Texte (auch solche, die der deutschen Mystik nahestehen). Vor allem aber wirkte sie im Bereich der Hagiographie. Konrad Kunze spricht von ihren „unermüdlichen hagiographischen Bemühungen"86. Ulla Williams und Werner WilliamsKrapp sehen dahinter ihre Absicht „eine geradezu enzyklopädisch konzipierte, auf mehrere Bände verteilte deutsche hagiographische Sammlung zustande zu bringen." ${ }^{87}$ „Regulas übersetzerische und redigierende Tätigkeit“, so Werner Williams-Krapp, „ist auf den Hausgebrauch und dessen spezifische Bedürfnisse ausgerichtet: Legendare, die breite Leserkreise anzusprechen vermochten, werden von ihr zu hagiographischen Erbauungsbüchern für ihre Mitschwestern umfunktioniert." Eine zentrale Stellung dabei hat das 'Buch der heiligen Mägde und Frauen', überliefert in einer um $1460 \mathrm{zu}$ datierenden Handschrift, ein Lob auf den Jungfrauenstand, das bei den Mitschwestern in einer Sammlung von 57 Lebensbildern heiliger Frauen die Entscheidung zum tugendsamen Leben vertiefen und festigen wollte. ${ }^{88}$ Das generell festgestellte enzyklopädisch zu nennende Vorgehen in der Hagiographie spiegelt sich in diesem Einzelprojekt: Dem Prolog des Buches, der Zweck und Ziel offen legt, ${ }^{89}$ folgt als erstes 'Lebensbild' dasjenige der Maria Magdalena, bestehend aus drei Texten:

${ }^{84}$ Zit. nach Schindele [Anm. 81], S. 225.

${ }^{85}$ Zit. nach Heinzer/Stamm [Anm. 81], S. 41.

${ }^{86}$ Kunze, Die Elsässische, Bd. II [Anm. 81], S. XXIV.

87 Williams/Williams-Krapp, Legenda aurea [Anm. 81], S. LVII.

${ }^{88}$ Williams-Krapp, Legendare [Anm. 81], S. 364.

${ }^{89}$ Dazu Kunze, Alemannische Legendare [Anm. 81], S. 30ff. und Feistner [Anm. 81]. 
Die Übersetzung aus der 'Legenda aurea' bildet den Schlusspunkt; dem vorausgehend die Gebete zu Maria Magdalena aus den 'Orationes et Meditationes' Anselms von Canterbury; eingeleitet aber wird der MariaMagdalena-Komplex von einem Text, den Kunze noch als „erste Legende" bezeichnete ${ }^{90}$, bei dem es sich freilich um die PseudoOrigenes-Homilie in einer sehr genauen Übersetzung handelt.

Regulas Arbeitstechnik generell zu beschreiben, ist nicht leicht. Sie kennt - und benutzt - verschiedene Verfahren. So haben Konrad Kunze, Ulla Williams und Werner Williams-Krapp an Regulas Bearbeitung einzelner Legenden der 'Legenda aurea' ihren souveränen, selbstbewussten und im Vergleich mit anderen Legendaren außerordentlich selbstständigen Umgang mit den Texten gezeigt und demonstriert, wie sie aus den verschiedensten Texten neue schuf, diese auch mit eigenen Fragen, Anmerkungen und Gedanken anreicherte. So gesehen trifft die Kennzeichnung bewusster Textänderungen in umfassender und stark ausgeprägter Weise zu. Dem steht im Umgang mit der einzelnen Vorlage und beim Übersetzen aber ein anderes Prinzip gegenüber. Williams und Williams-Krapp betonen mit Recht: „Die veritas latina war eine der Richtlinien ihrer Arbeit." ${ }^{1}$ Und auch Kunze hat festgestellt, Regula übersetzte „mit ihrem engen Anschluss an das Latein weitgehend nach dem Prinzip verbum de verbo." Ihre Selbstkritik (in worheit künd ich disz bisz her nit baz getütschet han, wie ermlich vnd vngesetzt es auch sy) beziehe sich womöglich auch darauf, dass ihre Art des Übersetzens ihrer eigenen Vorstellung von einem flüssigen deutschen Zieltext nicht gerecht wurde. ${ }^{92}$ Edith Feistner, die Regulas Übersetzung der Margarethenlegende untersucht hat, kommt sogar zu dem Ergebnis, diese sei „nichts weniger als eine 'laienhafte' Aneignung, sondern verrät ganz im Gegenteil sogar Tendenzen einer gelehrten Überkompensierung, die den volkssprachlichen Text geradezu 'lateinischer' erscheinen lassen als die Quelle". Feistner beschränkt ihre Untersuchung allerdings ausdrücklich auf das Gebiet der Legende. ${ }^{93}$

\footnotetext{
${ }^{90}$ Kunze, Alemannische Legendare [Anm. 81], S. 33.

91 Williams/Williams-Krapp, 'Legenda aurea' [Anm. 81], S. LVI.

${ }^{92}$ Kunze, Die Elsässische Bd. II [Anm. 81], S. XXXII.

93 Edith Feistner: Bausteine zu einer Übersetzungstypologie im Bezugssystem von Rezeptions- und Funktionsgeschichte der mittelalterlichen Heiligenlegende, WolframStudien XIV (1996), S. 171-184, hier S. 181-183.
} 
Mein kurzer Blick auf die Übersetzung der Pseudo-OrigenesHomilie kann dieses Urteil auch für die Heiligenpredigt bestätigen. Regula übersetzt im Vergleich mit den anderen Übersetzern am engsten nach dem Lateinischen. Dies ist in gewisser Weise bedauerlich, denn gerade die Bewertung der Szene am Grab durch eine Übersetzerin könnte unter gender-Aspekten natürlich aufschlussreich sein. Aber Regula verzichtet in diesem Text beinahe ganz auf Einschübe, Zusätze und Veränderungen. Nur einmal, als der lateinische Text Ct 8,6 zitiert, trägt sie die Quelle bei: als Salomon sprichet. ${ }^{94}$ So genau folgt Regula ihrer Vorlage, dass sie den Text nicht einmal bei den appellativen Stellen der Predigt auf ihr weibliches Publikum zuschneidet: Die fratres der lateinischen Anrede sind auch bei ihr zu Anfang und zum Ende des Textes $O$ liebe brüdere. ${ }^{95}$ Eine kleine Nuancierung kann aber doch festgestellt werden: Am Ende des Textes übersetzen die beiden anderen Übersetzer Heinrich Haller und Lienhart Peuger, dass vom Herrn eine „Kraft“, bzw. ein „Trost“ ausging, der alle gesunt machte. Regula lässt von inm dugend ${ }^{96}$ ausgehen und entspricht damit ihrem generellen Programm, wie es Williams-Krapp (s.o.) benannt hat, ihre Mitschwestern im t u g e n d samen Leben zu bestärken.

\section{IV.5.}

Beim letzten zu behandelnden Übersetzer kann ich mich kurz fassen: Der benediktinische Laienbruder Lienhart Peuger ${ }^{97}$, geboren um 1390, trat als früherer armiger im Jahr 1419 ins eben reformierte Stift Melk ein und legte 1420 als Laienbruder Profess ab. Er trat dort hervor als Verfasser eigener Reimpaarreden, war als Schreiber, Redaktor und Übersetzer tätig. Er legte als Hauptschreiber und Bibliothekar der Melker Laienbrüderbibliothek mit mehr als zwei Dutzend volkssprachlicher Handschriften den Grundstock für eine umfangreiche Bibliothek, die den

\footnotetext{
${ }^{94}$ Boxler [Anm. 48], S. 538, $10^{\text {vb. }}$

${ }^{95}$ Ebd., S. 537, $10^{\text {ra }}$ und 544, $15^{\text {vb }}$.

${ }^{96}$ Ebd., S. 544, $15^{\mathrm{vb}}$.

${ }^{97}$ Vgl. Freimut Löser: Peuger, Lienhart, in: ${ }^{2}$ VL Bd. 7, Sp. 534-537; Ders., Meister Eckhart in Melk. Studien zum Redaktor Lienhart Peuger. Mit einer Edition des Traktats Von der sel wirdichait vnd aigenschafft (TTG 48), Tübingen 1999.
} 
Mitbrüdern eine repräsentative Auswahl katechetischer, erbaulicher, scholastischer und mystischer Texte bot, darunter eben auch die Pseudo-Origenes-Homilie, die er relativ frei übersetzt (wie der Vergleich zeigt: am freiesten von allen deutschen Übersetzungen); er kürzt den Text punktuell und er schneidet inn auf sein Zielpublikum zu. Er inseriert den Text in seine große Predigtsammlung und stellt inn in eine Reihe von Osterpredigten des Nikolaus von Dinkelsbühl. ${ }^{98}$ Eine kurze Einleitung gibt an, dass Peuger auch weiß, worum es sich bei dem Text handelt: Dy predig ist genomen aws der ler origenis, wie maria magdalena den herren ihesum christum am ersten tag gesuecht hat. Aber Peuger teilt noch mehr mit: $d y$ predig hat genomen Maister Milicius von der awslegung Origenis. Unter allen Übersetzern ist Peuger der einzige, der den Ausgangstext der Pseudo-Origenes-Homilie und die Predigt Miličs kombiniert. Hier begegnet eine deutsche Übersetzung des lateinischen Prothemas des tschechischen Predigers. Auch der Grund, warum Peuger Milič heranzieht, lässt sich erkennen: Die vor red spricht: Maria stuend pey dem grab vnd waint iohannis $x x^{\circ}$. das ist wider $d y$ langen slaffer, dy predig vnd den dienst gots versawmen. Peuger folgt auch hier im Einzelnen dem Text Miličs, wie der Vergleich zeigt:

Das ist wider dy langen slaffer dy predig und den dienst gotes versawmen.

wann das ewangelium geit zu erchennen das maria magdalena früe zw grab cham da es noch tunkchel was vnd suecht irn maister als ein trewe Jüngrin wan sy het vor dy ewig weisheit reden hörn.
Contra diu dormientes et praedicationem negligentes evangelium testatur Mariam Magdalenam mane venisse cum adhuc tenebrae esset ad quaerendum magistrum tamquam bona disciplina audiens sapientiam ${ }^{9}$

${ }^{98}$ Melk, Stiftsbibliothek cod. 1865, f. $162^{\text {rb }}-178^{\text {ra }}$; hier f. $171^{\text {rb }}-176^{\text {rb }}$.

99 Lateinisches Incipit nach der Incipitsammlung von Franz Lackner: Iohannes Milicius Sermones „Gratiae Dei“ (http:// www.oeaw.ac.at/ksbm/serm/ioh mil.htm, T 28/5). 
Neben den bekannten deutschsprachigen Gebeten Miličs ist damit also auch eine deutsche Übersetzung einer seiner Predigten bekannt geworden. Dass diese gerade im Kloster Melk liegt, hat nichts Überraschendes. Die Beziehungen zwischen den an der Kirchenreform interessierten Kreisen in Prag und Wien, von wo aus sie auch Melk ergriffen, sind bekannt und gut erforscht. Das betrifft die äußere Überlieferung der Texte. Aber auch in den inhaltlichen Aussagen gibt es Affinitäten: In der Form Miličs und Peugers gewinnt die PseudoOrigenes-Predigt - zusätzlich zu ihrer tieferen Bedeutung - einen weiteren sehr konkreten Sinn, der sich den Melker Laienbrüdern als unmittelbare didaktische Nutzanwendung vermitteln ließ: Früh aufzustehen! Ähnlich wie bei Heinrich Haller, vielleicht aber noch deutlicher, wird der Text zum Mahnmal für die Reinheit des Glaubens:

Auch tue von dir allen irrsal des vnglaubens vnd rewt aws pöse pegier, das ihesus allain in deim hertzen wonen müg. ${ }^{100}$

Der Text ist in zweierlei Hinsicht interessant. Die Übersetzung transgrediert am Ende die Gender-Grenzen; sie fordert die 'weibliche' Eigenschaft der Trauer auch für die Brüder. Und: Der Text sucht, wenn er Trost sucht (und spendet), diesen Trost nicht in der Zeit und im gegenwärtigen Leben:

Dar vmb, mein aller liebst prüder, ein yeder vnter vns sol des weibs hitzige pegier nachuolgen vnd suehen ihesum in gantzer lieb vnserer tzäher. O mensch, lern von eins weibs persan vnd von einer sunterin, der ir sünt vergeben sind, vnd lern ze wainen von dem abschaiden gots [...]. Lern st(t ze sein in dem suehen ihesu. Lern niht ze fürchten widerzäme ding vnd lern an ihesum chainen tzeitleichen trast zw enphahen sunder ihesum selber. ${ }^{101}$

V.

${ }^{100}$ Melk, Stiftsbibliothek cod. 1865 , f. $175^{\mathrm{vb}}-176^{\mathrm{ra}}$.

${ }^{101}$ Ebd., f. $175^{\mathrm{vb}}$. 
Fazit: Der Text, in lateinischer Version im späten 12. oder frühen 13. Jahrhundert aus dem benediktinisch/zisterziensischen Milieu ausstrahlend, offenbart allein in seiner deutschen Version eine reiche Fülle von Lesarten und unterschiedlichen Realisationsformen. Ausgangspunkt ist das Changieren der Situation und der Heldin dieser Situation zwischen karfreitäglichem Leid und österlicher Auferstehungsfreude (die nur sehr zögerlich realisiert wird). Die zwischen Trauer und Trost (persönlichem und allgemeinem) gespannte Position des Ausgangstextes lässt sich auf Affekte, Affektverteidigung und Affektkritik ebenso beziehen wie auf religiös/geistliche Inhalte. Die Liebe und der Mut der Heldin eröffnen ebenso weitere Spielräume, wie die Diskussion des Trost-Gedankens und die Rolle des Predigers im Text. Zunächst ist eine apologetische Haltung erkennbar, die die Affekte Maria Magdalenas verteidigt; dabei verschieben die deutschen poetischen Bearbeitungen die Gewichte im Vergleich mit dem Quell-Text erheblich: In 'Der Saelden Hort' wird der Text dem wohl durch das Höfische geprägten Geschmack des Publikums angeglichen: Maria Magdalena erscheint als Heroin der Trauer; die äußeren Anzeichen der Trauer und ihre klare Erkennbarkeit werden fast wichtiger als der religiöse Diskurs. Es geht vorrangig um die Gebärden der Trauer. Trauer und Tod sind öffentliche Akte; sie folgen den 'Ritualen des Trauerns'. Nachvollzug des Leides wird zur Selbstkasteiung. Diese Verschiebung der Perspektive teilt auch Heinrichs von Neustadt 'Von Gottes Zukunft'; aber Heinrich unternimmt eine weitere 'Verschiebung': Der Teil des Werkes, der die Grabeszene aufgreift, thematisiert den Verlust des Trostes und die liebessehnsüchtige Trostlosigkeit der minnerin. Die Heldin wird zur Kämpferin gegen die Versuchungen des Teufels, so wie auch die Apostel zu gotes degen werden. Die didaktischen Möglichkeiten des Textes entfalten sich im Apell zur christlichen Tugendhaftigkeit. Die 'bairische Magdalenenklage' (ansonsten wenig bearbeitet) will dagegen in kurzen Einschüben den Zustand des Leides überwunden wissen und zielt damit stärker als der Ausgangstext auf die österliche Freude.

Die Predigt erschließt völlig andere Lesarten. Meister Eckhart, der die Ausgangsfassung zweimal bearbeitet (und dabei stark kürzt, sich aber auch stark an seiner Vorlage orientiert) verschiebt das Gesamtgefüge durch leichte Retuschierung in die von inm angezielte Richtung. Die Szene (und die Inszenierung der figürlichen Szene) am 
Grab tritt zurück hinter dem von Eckhart entdeckten Textsignal: Im Sinn der deutschen Dominikanermystik geht es hier vorrangig um das Verhältnis von Vielheit und Einheit: Wer den Herrn sucht, der trauert nicht nur um den toten Herrn, vielmehr wird inm die Kreatur zur Last (oder, wie Eckhart sagt, als Nichts erkannt). Es geht nicht mehr um die Verteidigung der Affekte, sondern um die Rückkehr in die Einheit.

Dieser Gedanke der Einheit wird durch den Franziskaner Marquard von Lindau vielleicht noch schärfer akzentuiert. Die Seele soll mit Gott vereint sein (und dies sunder mittel). Der Weg zur Einheit liegt (anders als im Ausgangstext, der die beiden Zugänge vereint und innen gemeinsam ihr Recht lässt) in der vita contemplativa (nicht in der vita activa).

Jan Milič von Kremsier, der 1374 gestorbene 'Vater der tschechischen Reformation' und Vorläufer von Jan Hus, kehrt dagegen in dem Text, den er für seine lateinische (auch in späterer übersetzter deutscher Version tradierte) Predigt weitgehend übernimmt, andere Aspekte in den Vordergrund: Christi Kreuzestod dient den Sündern. Die Abkehr von den Sünden und ein gottgefälliges Leben, das aktiv den frühen Gottesdienst (im litteralen und übertragenen Sinn) sucht - dafür steht Maria Magdalena als Exempel für Miličs Gemeinde.

Die deutsche Prosaübersetzungen des lateinischen Predigttextes aus dem 14. und 15. Jahrhundert wenden unterschiedliche Übersetzungsprinzipien an und stellen wieder andere Aspekte in den Vordergrund. Der höchst interessante Nürnberger 'Magdalenen-libellus' stellt dabei drei Gedanken in den Mittelpunkt: den Trost-Diskurs, die Gleichheit mit Gott und die hohe wirdikeit der Frau. Trost über den Tod beim Toten selbst zu suchen, ist ein fulminanter Irrtum; die Verzweiflung über den Tod ist das Hindernis des Vertrauens in die Auferstehung. Die adeliche frîheit teilt der Mensch mit Gott. Maria Magdalena wird zur Verkünderin der Auferstehung und dieser frîheit, die allen frauen ein ewig lob verdient. Der Kartäuser Heinrich Haller übersetzt den lateinischen Text bemerkenswert texttreu, wendet sich mit seiner Version an die Laienbrüder seiner Kartause und will konkret deren besserung erreichen. Die Zisterzienserin Regula gibt ebenfalls eine sehr genaue Übersetzung, die nicht einmal (obwohl sie für ihre Mitschwestern geschrieben ist) an der Anrede fratres rührt. So spricht Regula ihr weibliches Publikum mit $O$ liebe brüdere an, inseriert aber ihre 
Bearbeitung - als erstes 'Lebensbild' - ihrem 'Buch der heiligen Mägde und Frauen', das bei ihren Mitschwestern in einer Sammlung von 57 beispielhaften 'Lebensläufern' die Entscheidung zum tugendsamen Leben festigen will. Der benediktinische Laienbruder Peuger im reformierten Melk widmet seine freie Bearbeitung (aus Urtext und MiličPredigt) den Mitbrüdern und verstärkt die 'praktischen' Aspekte des Textes, der zum Apell für die Reinhaltung des Glaubens und für ein christliches Leben im Sinn der Klosterregel wird.

Alle Texte führen von ihrem gemeinsamen Ausgangspunkt jeweils weit über die Verteidigung des Affektes hinaus: Die Realisationsformen und Spielformen der Trauer eröffnen im geistlichen Bereich stets neue und immer wieder weiter führende 'Trostwelten'; von der 'Männlichkeit' der Trauernden bis zur einheitsstiftenden Funktion von trauernder Liebe, die hierin sogar jeden Trost überschreitet. 